\title{
Experiences with Applying Scenario-Based Approach to Refine Aircraft Stakeholder Requirements
}

\author{
Wenhao Zhu and Fuxing Tao
}

\begin{abstract}
The development of aircraft often involves much effort of requirements elicitation, definition, and review, and the initial version of these requirements are provided by aircraft stakeholders. In practice, as the initial stakeholders' requirements do not often meet the characteristics and attributes of INCOSE as good requirements, they might cause errors and difficulties to requirements verification and validation. Although the Easy Approach to Requirements Syntax (EARS) has been introduced to provide an easy method to define natural language requirements for years, the engineers and stakeholders still need to reach a shared comprehension of the requirements before defining them. The approach reported in this paper is to combine a scenario-based approach with templates as an elementary guidance for system engineers to refine requirements at the early stages of aircraft development project, so as to eliminate possible errors and problems caused by natural language and insufficient communication.
\end{abstract}

\footnotetext{
W. Zhu $(\bowtie) \cdot$ F. Tao

AVIC Digital Corporation Ltd., Building 19E, Compound A5, Shuguangxili, Chaoyang District, Beijing 100028, China

e-mail: zhuwh@avic-digital.com

F. Tao

e-mail: taofx@avic-digital.com
} 